\title{
Adverse Neonatal Outcomes and Its Associated Factors Among Mothers with Short and Recommended Interpregnancy Interval, in Awi Zone Public Hospitals, 2020.
}

Jemberu Chane Fetene

Jigjiga University

Amlaku Mulat

Bahir Dar University

kihinetu Gelaye Wudineh ( $\square$ kihinetugelaye031@gmail.com )

Bahir Dar universty

Tigist Wubet

Bahir Dar University

Research article

Keywords: Adverse neonatal outcomes, short IPI, recommended IPI, Ethiopia.

Posted Date: January 18th, 2021

DOl: https://doi.org/10.21203/rs.3.rs-146927/v1

License: (c) (1) This work is licensed under a Creative Commons Attribution 4.0 International License.

Read Full License 


\section{Abstract}

Background: Short interpregnancy interval is among modifiable risk factors for maternal and neonatal adverse outcomes for planned pregnancies. It is potentially associated with adverse neonatal outcomes which are known to have considerable public health significance. In Ethiopia neonatal mortality was found to be high according to recent mini Ethiopian Demographic Health Survey Report. More importantly information about adverse neonatal outcomes in relation to interpregnancy interval is poorly described yet in Ethiopia.

Methods: Institution based comparative cross-sectional study was conducted in Awi zone public hospitals. A total of 482 mothers ( 241 with short and 241 with recommended IPI) were selected. The data was collected by using systematic random sampling technique through pretested structured questionnaire and entered in to Epi data version 3.1 then exported to Statistical Package of Social Science version 23.0 for analysis. Chi-square and independent t-test were used to compare means. Bivariable and multivariable logistic regression analyses was employed to estimate the crude and adjusted odds ratio with a confidence interval of $95 \%$ and $P$ value of less than 0.05 considered statistically significant.

Result: Among a total of selected mothers with short and recommended Interpregnancy interval (IPI) response rate was $237(98.3 \%)$ and $238(98.7 \%)$ respectively. Proportion of adverse neonatal outcomes were higher among short IPI groups (37.1\%). Factors like, rural residence [AOR=6.9, 95\% $\mathrm{Cl}(3.32,14.59)]$, and Cesarean section delivery $[\mathrm{AOR}=3.4,95 \% \mathrm{Cl}(1.18,10.09)]$ were significantly associated with adverse neonatal outcomes in short IPI groups. Factors like rural residence [AOR=6.1, 95\% $\mathrm{Cl}(2.11,17.7)]$, unintended pregnancy $[A O R=5.3,95 \% \mathrm{Cl}(1.11,25.00)]$, rupture of membrane $[A O R=6.89,95 \% \mathrm{Cl}(2.54$, $18.65)]$ and induction of labor $[\mathrm{AOR}=13.4,95 \% \mathrm{Cl}(3.17,21.77)]$ were significantly associated in recommended IPI groups.

Conclusion: Urban residency and vaginal mode of delivery were significantly associated with less risk of adverse neonatal outcomes in both IPI groups. Whereas, intended pregnancy status, spontaneous labor initiation and absence of ROM before labor were protective for adverse neonatal outcomes in recommended IPI mothers. Provision of proper health service coverage at rural area and minimizing $\mathrm{C} / \mathrm{S}$ rate to reduce adverse neonatal outcome is recommended.

\section{Background}

Inter pregnancy period is an opportunity to address complications occurred during pregnancy, to assess a woman's mental and physical wellbeing and to optimize her health along her life time(1). Short Interpregnancy interval (IPI) is among modifiable risk factors for adverse neonatal outcomes for planned pregnancies(2). IPI is defined as the time elapsed between the woman's last delivery and the date of the last menstrual period for the index pregnancy $(1,3)$. 
There are existing recommendations that target optimization of women or couples health before conception. The CDC in 2006 and WHO in 2012 had recommended the need to implement highly recommended for good maternal and perinatal outcomes(4). Prevention of short interpregnancy intervals is a public health priority in the United States. Specifically the American Healthy People objectives call for a $10 \%$ reduction of pregnancies that occur within 18 months of a previous birth by 2020(5).

The health status of the couples, particularly of the woman, prior to pregnancy or conception and during the pregnancy period can adversely affect the outcome of the pregnancy(6). Short interpregnancy interval was found to be potentially associated with adverse neonatal outcomes including stillbirth, early neonatal mortality, preterm birth, Neonatal Intensive Care Unit (NICU) admission, low Apgar score and low birthweight (LBW) which are known to have considerable public health significance(7). Globally LBW occurs in $>20$ million newborns worldwide, which is a major contributor to perinatal mortality and up to $80 \%$ of neonatal mortality(8). Preterm birth complications are also the leading cause of deaths in the neonatal period(9).

Studies from developed countries showed that the risk of developing adverse neonatal outcomes to be higher in pregnancies with short IPI (10-12). Other reports from developed countries showed an increased risk of developing preterm birth and LBW eventhough they show insignificant association for other adverse neonatal outcomes(13). Similarly the effect of short IPI on adverse neonatal outcomes among developing countries was also supported by many literatures $(14,15)$.

In Ethiopia the perinatal mortality rate is relatively high among women with a pregnancy interval of less than 15 months ( 45 deaths per 1,000 pregnancies)(16). Despite; the availability of health facilities and improved health services, recent report from Ethiopian Demographic Health Survey (EDHS) determined that stagnant prevalence in neonatal mortality as compared to the previous 2016 EDHS report(17). Therefore this study aimed to compare pregnancy outcomes between short and recommended IPI and identify associated factors among women delivering at Awi zone public hospitals.

\section{Methods}

\section{Study area and period}

The study was conducted from February 15 to April 15, 2020, at public hospitals in Awi zone, Amhara region, North West Ethiopia, 2020. Awi zone is one of the 15 Zones in Amhara Region of Ethiopia. It is bordered on the west by Benishangul-Gumuz Region, on the north by Semien Gondar Zone and on the east by Mirab Gojjam. The administrative centre of Awi zone is Injibara; other towns include Chagni, and Dangila. Injibara is found $297 \mathrm{Km}$ from Adis ababa, Ethiopia. It has 11 woredas and a total of 5 public hospitals (dangla primary hospital, Injibara general hospital, Jawi primary hospital, and Gmjabet primary hospital and Chagni primary hospitals) and 447 health centers.

\section{Study Design And Population}


Institution based comparative cross-sectional study was conducted among all mothers who had at least one previous live birth and who gave their current birth in Awi zone public hospitals during the study period. Mothers whose charts were incomplete and whose current delivery was other than singleton were excluded from the study.

\section{Sample Size Determination}

Sample size was calculated using a double population proportion formula; assuming $22.2 \%$ proportion $\left(p_{1}\right)$ for the exposed and proportion $\left(p_{2}\right)$ for un exposed $11.3 \%$ based on a previous study which tried to show interpregnancy interval as a risk factor for preterm birth, with $95 \%$ level of confidence (z) and power of $80 \%$. By applying $10 \%$ of non-response rate the final sample size became 200 .

Sample size calculated using values from different previous literatures was determined by using Epi info version 7 by considering the following assumptions: confidence interval $(\mathrm{Cl}) 95 \%$, power $80 \%$, ratio 1:1 and non-response rate $10 \%$. The factors were taken from previous study conducted in Suhul Shire hospital, Gamo Gofa zone, North Wollo zone and Nigst Eleni hospital hosanna town[18-21](Table1).

Table 1 sample size calculation based on significant independent variables identified on literatures.

\begin{tabular}{|c|c|c|c|c|c|}
\hline Authors & Factor & $\begin{array}{l}\text { Prevalence of neonatal } \\
\text { adverse outcome } \\
P_{1} \text { (in exposed), } P_{2} \text { (in } \\
\text { unexposed) }\end{array}$ & Power & AOR & $\begin{array}{l}\text { Sample size with } \\
10 \% \text { non-response } \\
\text { rate }\end{array}$ \\
\hline $\begin{array}{l}\text { Adhena } \\
\text { et al. }\end{array}$ & residence & $\begin{array}{l}P_{1}=27.7 \% \\
P_{2}=16.2 \%\end{array}$ & $80 \%$ & $\begin{array}{l}1.643(0.93- \\
2.8)\end{array}$ & 482 \\
\hline $\begin{array}{l}\text { Feleke et } \\
\text { al. }\end{array}$ & $\begin{array}{l}\text { Occupational } \\
\text { status }\end{array}$ & $P_{1}=16.6 \%, p_{2}=1.4 \%$ & $80 \%$ & $\begin{array}{l}0.074 \\
(0.017 \\
0.324)\end{array}$ & 147 \\
\hline $\begin{array}{l}\text { Kasahun } \\
\text { et al. }\end{array}$ & Age & $\begin{array}{l}P_{1}=32.8 \% \\
P_{2}=20.5 \%\end{array}$ & $80 \%$ & $\begin{array}{l}0.5(0.20 \\
1.20)\end{array}$ & 480 \\
\hline $\begin{array}{l}\text { Abdo et } \\
\text { al. }\end{array}$ & $\begin{array}{l}\text { Marital } \\
\text { status }\end{array}$ & $\begin{array}{l}P_{1}=36 \% \\
P_{2}=20.6 \%\end{array}$ & $80 \%$ & $\begin{array}{l}0.47(0.25 \\
0.91)\end{array}$ & 321 \\
\hline
\end{tabular}

Since the sample size calculated from the factors gives the larger sample size, the sample size for this study was 482 .

\section{Data Collection Tools And Procedures}


Questionnaire was designed to meet the objective of this study and the study was based on interviewer administered questionnaire and chart review. The questionnaire was pretested on $5 \%(25)$ of the calculated sample size in durbete hospital. First, the English version of the questionnaire was prepared. Then it was translated to Amharic and Awingi version (local languages) and then translated back to English to check its consistency.

The questionnaire has three parts. The first include socio-demographic information such as age, educational level, and occupation, place of residence (urban and rural), the second part deal with maternal characteristics and the third neonatal outcome. By reviewing their chart sex of their infant, duration of their labor pain, mode of delivery, obstetric U/S estimate of their GA, APGAR score, birth weight of the newborn, were taken from their chart. Then in the postnatal ward just before their discharge mothers were interviewed.

\section{Measurement}

The outcome variable was measured as presence or absence of adverse neonatal outcomes.

Adverse neonatal outcome implies the presence of at least one or more of the following conditions in the current pregnancy. These include APGAR score less than 7, still birth, NICU admission, low birth weight, congenital anomaly, and preterm birth.

\section{Data Processing, Analysis And Interpretation}

The collected data were entered and cleaned using Epi data version 3.1, then exported to SPSS version 23 for analysis. Descriptive analysis, chi-square and independent t-test were conducted to summarize the data and the final result of the study was interpreted in the form of text, figures and tables. Binary logistic regression analysis was executed to see the association between independent and dependent variables.

All explanatory variables with $p<0.2$ in bivariable logistic regression were entered into multivariable logistic regression analysis and significant association was identified based on $p<0.05$ and odds ratio with $95 \% \mathrm{Cl}$ in multivariable logistic regression. The final model fitness was checked using HosmerLemeshow Goodness of Fit test $(p=0.519)$. Separate analysis was also done for mothers with both short and recommended IPI.

\section{Ethical Consideration}

After approval, ethical clearance was obtained from Institutional Review Board (IRB) of College of Medicine and Health sciences, Bahir Dar University. Then, official letter was written from College of Medicine and Health Sciences to each Awi zone public hospitals. The aim of the study was informed for each study participant, and the study participants had a right to refuse or discontinue participating in the 
research without any restriction. Finally informed written consent was obtained from each participant before data collection and confidentiality was assured.

\section{Results}

\section{Socio-demographic characteristics of participants}

Among the total of 482 mothers, 475 mothers were participated in the study which makes response rate of 98.5\%. Regarding IPI, 237 (49.9\%) were mothers with short IPI and 238 (50.1\%) were mothers with recommended IPI. The highest proportion, 88(37.1\%) short IPI mothers and 100 (42\%) recommended IPI mothers were in the age group of 25-29 years. The mean age of the mother was $30.95(S D \pm 5.46)$ among mothers with short IPI and 30.75 (SD \pm 4.6 ) among those mothers with recommended IPI. Almost all $227(95.8 \%)$ of mothers with short IPI and $237(99.6 \%)$ mothers with recommended IPI were married. More than half $133(56.1 \%)$ of mothers with short IPI and $129(54.2 \%)$ recommended IPI mothers were urban residents. Regarding the educational status of mothers, $84(35.4 \%)$ of mothers with short IPI and $103(43.3 \%)$ of mothers with recommended IPI didn't attended formal education. Concerning educational status of the husbands, $84(36.8 \%)$ husbands of mothers with short IPI and $94(39.5 \%)$ husbands of mothers with recommended IPI didn't attend formal education (Table 2). 
Table 2

sociodemographic characteristics of mothers with short and recommended IPI in Awi zone public hospitals, Northwest Ethiopia, 2020

\begin{tabular}{|c|c|c|c|}
\hline \multirow[t]{2}{*}{ Variables } & \multicolumn{2}{|c|}{ Adverse neonatal outcome } & \multirow[t]{2}{*}{ p-value } \\
\hline & Yes (\%) & No (\%) & \\
\hline \multicolumn{4}{|l|}{ Age } \\
\hline $20-24$ & $22(9.3 \%)$ & $10(4.2 \%)$ & \multirow[t]{4}{*}{0.045} \\
\hline $25-29$ & $88(37.2 \%)$ & $100(42 \%)$ & \\
\hline $30-34$ & $64(27 \%)$ & $71(29.8 \%)$ & \\
\hline 35 and above & $63(26.6 \%)$ & $57(24 \%)$ & \\
\hline \multicolumn{4}{|l|}{ Marital status } \\
\hline Married & $135(28.4 \%)$ & $329(69.3 \%)$ & \multirow[t]{2}{*}{0.430} \\
\hline Unmarried* & $2(0.4 \%)$ & $9(1.9 \%)$ & \\
\hline \multicolumn{4}{|l|}{ Religion } \\
\hline Orthodox & $116(24.4 \%)$ & $276(58.1 \%)$ & \multirow[t]{3}{*}{0.508} \\
\hline Muslim & $19(4 \%)$ & $51(10.7 \%)$ & \\
\hline Protestant & $2(0.4 \%)$ & $11(2.3 \%)$ & \\
\hline \multicolumn{4}{|c|}{ Educational status of the mother } \\
\hline No formal education & $56(11.7 \%)$ & $131(27.6 \%)$ & \multirow[t]{3}{*}{0.874} \\
\hline Primary & $45(9.5 \%)$ & $111(23.4 \%)$ & \\
\hline Secondary and above & $36(7.6 \%)$ & $96(20.2 \%)$ & \\
\hline \multicolumn{4}{|l|}{ Occupation of the mother } \\
\hline Farmer & $54(11.4 \%)$ & $118(24.8 \%)$ & \multirow[t]{4}{*}{0.590} \\
\hline House wife & $52(10.9 \%)$ & $151(31.7 \%)$ & \\
\hline Governmental employee & $23(4.8 \%)$ & $49(10.3 \%)$ & \\
\hline Merchant & $10(1.7 \%)$ & $20(4.2 \%)$ & \\
\hline \multicolumn{4}{|c|}{ Husbands educational status } \\
\hline No formal education & $77(32.4 \%)$ & $91(38.2 \%)$ & \multirow[t]{3}{*}{0.256} \\
\hline Primary & $58(24.5 \%)$ & $62(26.1 \%)$ & \\
\hline Secondary and above & $96(40.1 \%)$ & $74(31.1 \%)$ & \\
\hline
\end{tabular}




\begin{tabular}{|c|c|c|c|}
\hline \multirow[t]{2}{*}{ Variables } & \multicolumn{2}{|c|}{ Adverse neonatal outcome } & \multirow[t]{2}{*}{ p-value } \\
\hline & Yes (\%) & No (\%) & \\
\hline \multicolumn{4}{|l|}{ Residence } \\
\hline Rural & $94(19.9 \%)$ & $120(25.3 \%)$ & \multirow[t]{2}{*}{$<0.001$} \\
\hline Urban & $43(9.1 \%)$ & $218(45.9 \%)$ & \\
\hline \multicolumn{4}{|c|}{ Husbands occupational status } \\
\hline Farmer & $11(2.3 \%)$ & $27(5.7 \%)$ & \multirow[t]{4}{*}{0.942} \\
\hline Daily labourer & $60(12.6 \%)$ & $144(30.3 \%)$ & \\
\hline Government employee & $30(6.3 \%)$ & $69(14.5 \%)$ & \\
\hline Merchant & $36(7.6 \%)$ & 98 (20.6\%) & \\
\hline
\end{tabular}

\section{Obstetric Characteristics Of Women With Short And Recommended Ipi}

The current pregnancy was planned wanted and supported in $196(82.7 \%)$ and $217(91.2 \%)$ of mothers with short and recommended IPI respectively. In more than three fourth $202(85.2 \%)$ and $186(78.2 \%)$ of mothers with short and recommended IPI respectively the presentation of the fetus was vertex. In almost all, 225(94.9\%) and 225(94.9\%) of mothers with short and recommended IPI respectively, the current pregnancy was completed at term GA. Labor started spontaneously in 231(97.1\%) of mothers with short IPI and 220(92.4\%) of mothers with recommended IPI. Nearly all, 236(99.6\%) and 234(98.3\%) of mothers with short and recommended IPI respectively had ANC follow up and $29(12.2 \%)$ of mothers from short IPI group and $33(13.9 \%)$ of those mothers from recommended IPI group started ANC late.

During their current pregnancy $6(2.5 \%)$ mothers with short IPI and $14(5.9 \%)$ of those mothers with recommended IPI faced obstetric complication. It was hypertensive disorder which accounts more $5(83.3 \%)$ and $12(85.7 \%)$ among short and recommended IPI mothers respectively. The overall proportion of ROM was $67(14.1 \%)$ and was prolonged in $26(38.8 \%)$ of cases. The mean duration of ROM was 7.66 $(S D \pm 5.09)$. In $31(6.5 \%)$ of cases duration of labor took $12 \mathrm{hr}$ and above, while the mean duration of labor was $6.48(\mathrm{SD} \pm 2.48)$ (Table 3 ). 
Table 3

obstetric characteristics of mothers with short and recommended

IPI in Awi zone public hospitals, Northwest Ethiopia, 2020.

\begin{tabular}{|c|c|c|c|}
\hline \multirow[t]{2}{*}{ Variable } & \multicolumn{2}{|c|}{ Adverse neonatal outcome } & \multirow[t]{2}{*}{$\mathrm{p}$-value $\left(\mathrm{x}^{2}\right)$} \\
\hline & Yes (\%) & No (\%) & \\
\hline \multicolumn{4}{|l|}{ Gravidity } \\
\hline $2-5$ & $54(22.8 \%)$ & $38(16 \%)$ & \multirow[t]{2}{*}{0.613} \\
\hline 5 and above & $34(14.3 \%)$ & $11(4.6 \%)$ & \\
\hline \multicolumn{4}{|l|}{ Parity } \\
\hline $2-5$ & $55(23.2 \%)$ & $37(15.5 \%)$ & \multirow[t]{2}{*}{0.76} \\
\hline 5 and above & $33(13.9 \%)$ & $12(5 \%)$ & \\
\hline \multicolumn{4}{|c|}{ Pregnancy status } \\
\hline Intended & $108(22.7 \%)$ & $313(65.9 \%)$ & \multirow[t]{2}{*}{$<0.001$} \\
\hline Unintended & $29(6.1 \%)$ & $25(5.26 \%)$ & \\
\hline \multicolumn{4}{|l|}{ ANC } \\
\hline Four and above & $98(20.6 \%)$ & $245(51.6 \%)$ & \multirow[t]{2}{*}{0.834} \\
\hline Less than four & $39(8.2 \%)$ & $93(19.1 \%)$ & \\
\hline \multicolumn{4}{|c|}{ Tetanus toxoid (TT) vaccinated } \\
\hline Yes & $133(28 \%)$ & $333(70.1 \%)$ & \multirow[t]{2}{*}{0.297} \\
\hline No & $4(0.8 \%)$ & $5(1.1 \%)$ & \\
\hline \multicolumn{4}{|c|}{ Complication during pregnancy } \\
\hline No & $124(26.1 \%)$ & $331(69.7 \%)$ & \multirow[t]{2}{*}{$<0.001$} \\
\hline Yes & $13(2.7 \%)$ & $7(1.5 \%)$ & \\
\hline \multicolumn{4}{|l|}{ ROM } \\
\hline Yes & $39(8.2 \%)$ & $28(5.9 \%)$ & \multirow[t]{2}{*}{$<0.001$} \\
\hline No & $98(20.6 \%)$ & $310(65.3 \%)$ & \\
\hline \multicolumn{4}{|l|}{ IPI } \\
\hline Short & 88 & 149 & \multirow[t]{2}{*}{$<0.001$} \\
\hline Recommended & 49 & 189 & \\
\hline
\end{tabular}




\begin{tabular}{|llll|}
\hline Variable & \multicolumn{2}{l}{ Adverse neonatal outcome } & p-value $\left(\mathbf{x}^{2}\right)$ \\
\cline { 2 - 3 } & Yes (\%) & No (\%) & \\
\hline Less than 8hr & $1(3 \%)$ & $10(29.4 \%)$ & 0.312 \\
8hr and above & $2(6 \%)$ & $5(14.7 \%)$ & \\
\cline { 2 - 3 } Mode of delivery & & \\
\hline Vaginal & $116(24.4)$ & $316(66.5)$ & 0.002 \\
C/S & $21(4.4 \%)$ & $22(4.6 \%)$ & \\
\hline
\end{tabular}

\subsection{Factors associated with adverse neonatal outcomes among mothers with short IPI}

Three models were fitted to assess factors in relation to adverse neonatal outcomes. The first model was fitted to identify factors associated with adverse neonatal outcomes among mothers with short IPI.

Variables such as residence, mode of delivery and time of initiation of ANC follow-up were found to have significant association with adverse neonatal outcomes. The odds of Mothers from rural area to develop adverse neonatal outcomes were 6.9 times $(A O R=6.9,95 \% \mathrm{Cl}=3.32,14.59)$ higher than those mothers from urban area. Mothers who deliver through $\mathrm{C} / \mathrm{S}$ were 3.21 times $(\mathrm{AOR}=3.21,95 \% \mathrm{Cl}=1.08,9.50)$ more likely to have babies with adverse neonatal outcomes than their counterparts (Table 4). 
Table 4

multivariable analysis of adverse neonatal outcomes among mothers with short IPI in Awi zone public hospitals, Northwest Ethiopia, 2020

\begin{tabular}{|c|c|c|c|c|c|}
\hline \multirow[t]{2}{*}{ Variables } & \multicolumn{5}{|c|}{ Adverse neonatal outcomes } \\
\hline & Yes & No & COR95\%Cl & AOR95\%Cl & $\begin{array}{l}\text { p- } \\
\text { value }\end{array}$ \\
\hline \multicolumn{6}{|l|}{ Age group } \\
\hline $20-24$ & $8(3.4 \%)$ & $14(5.9 \%)$ & $0.7(0.27,2.02)$ & $1.53(0.41,5.71)$ & 0.525 \\
\hline $25-29$ & $31(13.1 \%)$ & $56(23.6)$ & $0.72(0.37,1.39)$ & $2.02(0.8,5.07)$ & 0.132 \\
\hline $30-34$ & $20(8.4 \%)$ & 41 (17.3\%) & $0.64(0.3,1.31)$ & $1.0(0.41,2.44)$ & 0.988 \\
\hline 35 and above & $29(12.2 \%)$ & $38(16.0 \%)$ & 1 & 1 & \\
\hline \multicolumn{6}{|l|}{ Residence } \\
\hline \multirow{2}{*}{$\begin{array}{l}\text { Rural } \\
\text { Urban }\end{array}$} & $61(25.7 \%)$ & $45(19 \%)$ & $5.22(3.01,13.6)$ & $6.9(3.32,14.59)$ & $\begin{array}{l}<.001 \\
0.001\end{array}$ \\
\hline & $27(11.4 \%)$ & $\begin{array}{l}104 \\
\text { (43.9\%) }\end{array}$ & 1 & 1 & \\
\hline \multicolumn{6}{|c|}{ Educational status of the mother } \\
\hline No formal education & $42(17.7 \%)$ & $42(17.7 \%)$ & $1.01(0.53,1.73)$ & & 0.348 \\
\hline Primary & $29(12.2 \%)$ & $52(21.9 \%)$ & $\begin{array}{l}0.87(0.45 \\
1.70)\end{array}$ & & 0.293 \\
\hline $\begin{array}{l}\text { Secondary and } \\
\text { above }\end{array}$ & $32(12.7 \%)$ & $40(16.5 \%)$ & 1 & & 0.323 \\
\hline \multicolumn{6}{|l|}{ Sex of new born } \\
\hline \multirow{2}{*}{$\begin{array}{l}\text { Male } \\
\text { Female }\end{array}$} & $64(13.5 \%)$ & $\begin{array}{l}138 \\
(29.1 \%)\end{array}$ & $1.27(0.5,1.18)$ & $1.18(0.62,1.26)$ & 0.598 \\
\hline & $73(15.4 \%)$ & $\begin{array}{l}200 \\
(42.1 \%)\end{array}$ & 1 & 1 & \\
\hline \multicolumn{6}{|l|}{ ANC } \\
\hline Less than 4 & $20(8.4 \%)$ & $44(18.6 \%)$ & $0.8(0.63,2.13)$ & $1.2(0.55,2.90)$ & 0.563 \\
\hline 4 and above & $68(28.7 \%)$ & $\begin{array}{l}105 \\
(44.3 \%)\end{array}$ & 1 & 1 & \\
\hline \multicolumn{6}{|c|}{ RH status of the mother } \\
\hline negative & $11(4.6 \%)$ & $11(4.6 \%)$ & $1.7(0.7,4.3)$ & $2.84(0.94,8.58)$ & 0.064 \\
\hline Positive & 77 (32.5\%) & $\begin{array}{l}138 \\
(58.2 \%)\end{array}$ & 1 & 1 & \\
\hline
\end{tabular}




\begin{tabular}{|c|c|c|c|c|c|}
\hline \multirow[t]{2}{*}{ Variables } & \multicolumn{5}{|c|}{ Adverse neonatal outcomes } \\
\hline & Yes & No & COR95\%Cl & AOR95\%Cl & $\begin{array}{l}\text { p- } \\
\text { value }\end{array}$ \\
\hline \multicolumn{6}{|c|}{ Complication during pregnancy } \\
\hline \multirow{2}{*}{$\begin{array}{l}\text { Yes } \\
\text { No }\end{array}$} & $5(1.1 \%)$ & $15(3.2 \%)$ & $4.2(0.51,33.9)$ & $\begin{array}{l}1.98 \\
(0.24,16.01)\end{array}$ & 0.520 \\
\hline & $\begin{array}{l}139 \\
(29.3 \%)\end{array}$ & $\begin{array}{l}316 \\
(66.5 \%)\end{array}$ & 1 & 1 & \\
\hline \multicolumn{6}{|l|}{ ROM } \\
\hline Yes & $21(8.9 \%)$ & $12(5.1 \%)$ & $3.5(1.61,7.70)$ & $2.22(0.89,5.56)$ & 0.087 \\
\hline No & $67(28.35)$ & $\begin{array}{l}137 \\
(57.8 \%)\end{array}$ & 1 & 1 & \\
\hline \multicolumn{6}{|l|}{ Mode of delivery } \\
\hline $\mathrm{C} / \mathrm{S}$ & $9(3.8 \%)$ & $13(5.5 \%)$ & $2.4(1.13,5.42)$ & $3.21(1.08,9.50)$ & 0.035 \\
\hline Vaginal delivery & 75 (31.6\%) & $\begin{array}{l}140 \\
(59.1 \%)\end{array}$ & 1 & 1 & \\
\hline \multicolumn{6}{|l|}{ Pregnancy status } \\
\hline Un intended & $21(8.9 \%)$ & $20(8.4 \%)$ & 1 & 1 & \\
\hline Intended & $67(28.3 \%))$ & $\begin{array}{l}129 \\
(54.4 \%)\end{array}$ & $2.25(1.13,4.48)$ & $1.68(0.75,3.78)$ & 0.206 \\
\hline \multicolumn{6}{|l|}{ ANC initiation } \\
\hline Late & $5(2.1 \%)$ & $23(9.7 \%)$ & $0.33(0.12,0.91)$ & $0.61(0.22,1.21)$ & 0.008 \\
\hline Early & $83(35 \%)$ & $\begin{array}{l}126 \\
(53.2 \%)\end{array}$ & 1 & 1 & \\
\hline
\end{tabular}

Factors associated with adverse neonatal outcomes among mothers with recommended IPI

The second model was fitted to assess factors associated with adverse neonatal outcomes among mothers with recommended IPI. Variables like residence, pregnancy status, presence of ROM before labor and labor status were found to have a significant association with adverse neonatal outcomes among mothers with recommended IPI. The odds of delivering babies with adverse neonatal outcome among rural mothers was 6.1 times $(A O R=6.1,95 \% \mathrm{Cl}=2.11,17.7)$ higher than their counterparts. The odds of delivering babies with adverse outcome among mothers whose pregnancy was unintended was found to be 5.3 times $(A O R=5.3,95 \% \mathrm{Cl}=1.11,25.00)$ higher than their counterparts. Similarly mothers who had induction of labor were 13.4 times $(A O R=13.4,95 \% \mathrm{Cl}=3.17,21.77)$ more likely to deliver babies with adverse neonatal outcomes than those whose labor start spontanously. The odds of having babies with 
adverse neonatal outcomes in mothers who had ROM before labor was 6.89 times $(A O R=6.89,95 \% \mathrm{Cl}=$ 2.54) higher than their counterparts (Table 5) 
Table 5

distribution of adverse neonatal outcomes among women with recommended IPI in Awi zone public hospitals, Northwest Ethiopia, 2020.

\begin{tabular}{|c|c|c|c|c|c|}
\hline \multirow[t]{2}{*}{ Variables } & \multicolumn{5}{|c|}{ Adverse neonatal outcomes } \\
\hline & Yes & No & COR 95\%Cl & AOR 95\%Cl & $\begin{array}{l}\text { p- } \\
\text { value }\end{array}$ \\
\hline \multicolumn{6}{|l|}{ Age group } \\
\hline $\begin{array}{l}20-24 \\
25-29\end{array}$ & $4(1.7 \%)$ & $6(2.5 \%)$ & $\begin{array}{l}1.76 \\
(0.44,7.03)\end{array}$ & $1.02(0.13,8.09)$ & 0.980 \\
\hline $30-34$ & $21(8.8 \%)$ & 77 (32.4\%) & $\begin{array}{l}0.72 \\
(0.34,1.51)\end{array}$ & $0.61(0.18,1.99)$ & 0.607 \\
\hline \multirow[t]{2}{*}{35 and above } & $7(2.9 \%)$ & $61(18.9 \%)$ & $0.3(0.11,0.99)$ & $0.32(0.08,1.20)$ & 0.093 \\
\hline & $17(7.1 \%)$ & 45 (18.9\%) & 1 & 1 & \\
\hline \multicolumn{6}{|l|}{ Residence } \\
\hline \multirow{2}{*}{$\begin{array}{l}\text { Rural } \\
\text { Urban }\end{array}$} & $\begin{array}{l}33 \\
(13.9 \%)\end{array}$ & 75 (31.5\%) & $\begin{array}{l}3.13 \\
(1.68,16.2)\end{array}$ & $6.1(2.11,17.7)$ & $\begin{array}{l}<.001 \\
0.00\end{array}$ \\
\hline & $16(6.7 \%)$ & $\begin{array}{l}114 \\
\text { (47.9\%) }\end{array}$ & 1 & 1 & \\
\hline
\end{tabular}

\section{Educational status of the mother}

$\begin{array}{llll}\text { No formal education } \quad 23(9.7 \%) & 78(32.8 \%) & 1.25 \\ & & \end{array}$

Primary

$17(7.1 \%) \quad 59(24.8 \%)$

0.69

$(0.18,4.05)$

Secondary and above $\quad 9(3.8 \%) \quad 52(21.8 \%) \quad 1$

Occupational status of participant

House wife

Farmer

Governmental

employee

Merchant

Sex of new born

Male

Female

$\begin{array}{lll}33 & 80(33.8 \%) & 1\end{array}$

$32 \quad 45(19 \%) \quad 1.12$

$(0.56,2.25)$

0.738

0.619

$(0.28,2.13)$

0.46

0.337

\begin{tabular}{lll}
$17(7.2 \%)$ & $20(8.4 \%)$ & $\begin{array}{l}0.77 \\
(0.28,2.13)\end{array}$ \\
& & $\begin{array}{l}0.46 \\
(0.09,2.21)\end{array}$ \\
\hline & $4(1.7 \%)$ &
\end{tabular}




\begin{tabular}{|c|c|c|c|c|c|}
\hline \multirow[t]{2}{*}{ Variables } & \multicolumn{5}{|c|}{ Adverse neonatal outcomes } \\
\hline & Yes & No & COR 95\%Cl & AOR 95\%Cl & $\begin{array}{l}\text { p- } \\
\text { value }\end{array}$ \\
\hline & $\begin{array}{l}25 \\
(10.5 \%)\end{array}$ & $\begin{array}{l}106 \\
(44.5 \%)\end{array}$ & 1 & 1 & \\
\hline \multicolumn{6}{|l|}{ ANC } \\
\hline Less than 4 & $19(8.0 \%)$ & $49(20.6 \%)$ & $1.81(0.71,4.2)$ & $1.53(0.63,3.70)$ & 0.592 \\
\hline 4 and above & $\begin{array}{l}30 \\
(12.6 \%)\end{array}$ & $\begin{array}{l}140 \\
(58.8 \%)\end{array}$ & 1 & 1 & \\
\hline \multicolumn{6}{|c|}{$\mathrm{RH}$ status of the mother } \\
\hline Negative & $9(3.8 \%)$ & $20(8.4 \%)$ & $1.8(0.81,4.48)$ & $1.43(0.47,4.39)$ & 0.904 \\
\hline Positive & $\begin{array}{l}40 \\
(16.8 \%)\end{array}$ & $169(71 \%)$ & 1 & 1 & \\
\hline \multicolumn{6}{|l|}{ ROM } \\
\hline \multirow{2}{*}{$\begin{array}{l}\text { Yes } \\
\text { No }\end{array}$} & $18(7.6 \%)$ & $16(6.7 \%)$ & $6.27(2.3,17.8)$ & $6.89(2.54,18.6)$ & $\begin{array}{l}<.001 \\
0.001\end{array}$ \\
\hline & $\begin{array}{l}31 \\
(13.0 \%)\end{array}$ & $\begin{array}{l}173 \\
(72.7 \%)\end{array}$ & 1 & 1 & \\
\hline \multicolumn{6}{|l|}{ Labor status } \\
\hline \multirow{2}{*}{$\begin{array}{l}\text { Induced } \\
\text { Spontaneous }\end{array}$} & $12(5 \%)$ & $6(2.5 \%)$ & $10(3.4,22.0)$ & $\begin{array}{l}13.4 \\
(3.17,21.77)\end{array}$ & $\begin{array}{l}<.001 \\
0.00\end{array}$ \\
\hline & $\begin{array}{l}37 \\
(15.5 \%)\end{array}$ & $\begin{array}{l}183 \\
(76.9 \%)\end{array}$ & 1 & 1 & \\
\hline \multicolumn{6}{|l|}{ Mode of delivery } \\
\hline \multirow{2}{*}{$\begin{array}{l}\text { C/S } \\
\text { Vaginal delivery }\end{array}$} & $8(3.4 \%)$ & $13(5.5 \%)$ & $2.6(0.78,8.89)$ & $\begin{array}{l}2.96 \\
(0.917,9.56)\end{array}$ & 0.070 \\
\hline & $\begin{array}{l}41 \\
(17.2 \%)\end{array}$ & $\begin{array}{l}176 \\
(73.9 \%)\end{array}$ & 1 & 1 & \\
\hline \multicolumn{6}{|l|}{ Pregnancy status } \\
\hline Un intended & $8(3.4 \%)$ & $5(2.1 \%)$ & $7.1(1.02,24.1)$ & $5.3(1.11,25.00)$ & 0.032 \\
\hline Intended & $\begin{array}{l}41 \\
\text { (17.2\%) }\end{array}$ & $\begin{array}{l}184 \\
(77.3 \%)\end{array}$ & 1 & 1 & \\
\hline
\end{tabular}

Factors associated with adverse neonatal outcomes among mothers with short and recommended IPI A full model was developed to assess factors associated with adverse neonatal outcomes among mothers with short and recommended IPI. In this model variables like residence, IPI, presence of ROM, 
labor status and mode of delivery were found to be significantly associated with adverse neonatal outcomes regardless of the IPI. Mothers with IPI of less than 24 month were 3.39 times (AOR = 3.39, $95 \% \mathrm{Cl}=2.02,5.7)$ more likely to develop adverse neonatal outcome than their counter parts. Rural resident mothers were 6.3 times $(A O R=6.3,95 \% \mathrm{Cl}=3.52)$ more likely to give birth of newborn with adverse neonatal outcome compared to mothers from the urban residency. Similarly mothers with ROM were also found to be 6.2 times $(A O R=6.2,95 \% \mathrm{Cl}=3.01,12.8)$ more likely to deliver newborn with adverse neonatal outcomes than their counterparts. In this study, Mothers who had induction of labor were 3.88 times $(A O R=3.88,95 \% \mathrm{Cl}=1.14,10.71)$ more likely to deliver newborn with adverse neonatal outcome as compared to their counterparts. Mothers who gave birth through $\mathrm{C} / \mathrm{S}$ were 2.4 times (AOR = $2.4,95 \% \mathrm{Cl}=1.17,5.2)$ more likely to have adverse neonatal outcomes than those with vaginal deliveries (Table 6). 
Table 6

multivariable analysis of adverse neonatal outcomes for mothers with both short and recommended IPI, in Awi zone public hospitals, Northwest Ethiopia, 2020.

\begin{tabular}{|c|c|c|c|c|c|}
\hline \multirow[t]{2}{*}{ Variables } & \multicolumn{5}{|c|}{ Adverse neonatal outcomes } \\
\hline & Yes & No & COR95\%Cl & AOR95\%Cl & p-value \\
\hline \multicolumn{6}{|l|}{ Age group } \\
\hline $20-24$ & $12(2.5 \%)$ & $20(4.2 \%)$ & $1.08(0.2,3.02)$ & $1.1(0.37,3.2)$ & 0.324 \\
\hline $25-29$ & $52(10.9 \%)$ & $133(28.0)$ & $0.7(0.30,2.18)$ & $1.05(0.52,2.13)$ & 0.89 \\
\hline $30-34$ & $27(5.7 \%)$ & $102(21.3 \%)$ & $0.47(0.15,1.13)$ & $0.6(0.29,1.21)$ & 0.399 \\
\hline 35 and above & $46(9.7 \%)$ & $83(17.5 \%)$ & 1 & 1 & \\
\hline \multicolumn{6}{|l|}{ Residence } \\
\hline Rural & $94(25.3 \%)$ & $120(25.3 \%)$ & $4(3.3,10.72)$ & $6.3(3.52,11.6)$ & $<0.001$ \\
\hline Urban & $43(9.1 \%)$ & $218(45.9 \%)$ & 1 & 1 & \\
\hline \multicolumn{6}{|l|}{ IPI } \\
\hline Short IPI & $88(18.5 \%)$ & $149(31.4 \%)$ & $2.27(1.8,4.01)$ & $3.39(2.02,5.7)$ & $<0.001$ \\
\hline Recommended IPI & 49 (10.3\%) & $189(39.8 \%)$ & 1 & 1 & \\
\hline \multicolumn{6}{|l|}{ Sex of new born } \\
\hline Male & $64(13.5 \%)$ & $138(29.1 \%)$ & $1.27(0.15,1.88)$ & $1.01(0.59,1.53)$ & 0.948 \\
\hline Female & $73(15.4 \%)$ & $200(42.1 \%)$ & 1 & 1 & \\
\hline \multicolumn{6}{|l|}{ Parity } \\
\hline 5 and above & $45(20.6 \%)$ & $84(17.7 \%)$ & $3.2(0.59,5.8)$ & $2.1(0.75,6.06)$ & 0.74 \\
\hline $2-5$ & 92 (19.4\%) & 254 (53.5\%) & 1 & 1 & \\
\hline \multicolumn{6}{|l|}{ ANC } \\
\hline Less than 4 & $39(8.2 \%)$ & 93 (19.6\%) & $1.04(0.37,1.36)$ & & 0.968 \\
\hline 4 and above & $98(20.6 \%)$ & $245(51.6 \%)$ & 1 & & \\
\hline \multicolumn{6}{|l|}{ ROM } \\
\hline Yes & 45 (9.5\%) & $22(4.6 \%)$ & $5.60(3.22,9.77)$ & $6.2(3.01,12.8)$ & $<0.001$ \\
\hline No & 109 (22.9\%) & 299 (62.9\%) & 1 & 1 & \\
\hline \multicolumn{6}{|l|}{ Mode of delivery } \\
\hline $\mathrm{C} / \mathrm{S}$ & $21(4.4 \%)$ & $22(4.6 \%)$ & $2.6(1.13,5.42)$ & $2.4(1.17,5.2)$ & 0.025 \\
\hline
\end{tabular}




\begin{tabular}{|c|c|c|c|c|c|}
\hline \multirow[t]{2}{*}{ Vạginaldeselivery } & \multicolumn{5}{|c|}{ Adverse neonatal outcomes } \\
\hline & Yes & No & COR95\%Cl & AOR95\%Cl & p-value \\
\hline & $116(24.4 \%)$ & $316(66.5 \%)$ & 1 & 1 & \\
\hline \multicolumn{6}{|l|}{ Labor status } \\
\hline Induced & $14(2.9 \%)$ & $10(2.1 \%)$ & $3.72(1.61,8.62)$ & $3.88(1.14,10.71)$ & 0.009 \\
\hline Spontaneous & $123(25.9 \%)$ & $328(69.1 \%)$ & 1 & 1 & \\
\hline \multicolumn{6}{|l|}{ Pregnancy status } \\
\hline Un intended & $29(6.1 \%)$ & $25(5.3 \%)$ & $3.3(1.8,5.58)$ & $1.93(0.93,3.89)$ & 0.078 \\
\hline Intended & $108(22.7 \%)$ & $313(65.9 \%)$ & 1 & 1 & \\
\hline \multicolumn{6}{|c|}{ RH status of the mother } \\
\hline Positive & $20(4.2 \%)$ & $31(6.5 \%)$ & $1.69(0.92,3.088)$ & $1.86(0.88,3.95)$ & 0.103 \\
\hline Negative & 117 (24.6\%) & 307 (64.6\%) & 1 & 1 & \\
\hline
\end{tabular}

\section{Discussion}

The overall proportion of adverse neonatal outcome in this study was $28.8 \%$. This result was found to be consistent with studies done in East Gojjam(22) and north Wollo(23), 31.7\% and 31.8\% respectively. Whereas the overall prevalence in this study was found to be higher than a study from Gondar specialized teaching hospital, 23\%(24). This might be because the study of Gondar specialized teaching hospital estimate proportions only for stillbirth, preterm birth and LBW, they didn't include other adverse outcomes like APGAR score $<7$, NICU admission nor major congenital anomalies.

Waiting at least 24 months before trying to become pregnant after a live birth is highly recommended as it helps to avoid the risk of developing poor neonatal and infant health outcome (25). The finding of this study also supports this recommendation, rates of adverse neonatal outcomes were found to have a significant association with short IPI. This result is also supported by a case-control study from northwest Ethiopia(26) and another cohort study from southern Ethiopia(27). It was also consistent with studies from Sudan and Qatar(14, 28).

This study also revealed that, unintended pregnancy status was significantly associated with adverse neonatal outcomes among mothers with short IPI. This result was consistent with a study done in southern Ethiopia that showed unintended pregnancy status to be associated with risk of adverse neonatal outcomes(29). This is because those mothers with unintended pregnancy status are less likely to seek care from health institutions $(30,31)$ which might alter maternal use of antenatal care services, subsequent poor labor and delivery care and in adequate neonatal care. 
Maternal residence was found significantly associated with adverse neonatal outcomes. In this study rural residents were found more likely to have adverse neonatal outcome than urban residents. This finding was consistent with a report from Nigst Eleni hospital in hosanna(18), North Wollo(23), Gamo Gofa(20) and Mekelle(32) which also showed rural residents to have significantly higher risk of developing adverse neonatal outcomes. This might be due to distance naturally prevents mothers from doing so even if they are knowledgeable of the benefits of antenatal care services but deprives them the opportunity for early identification and management of pregnancy related problems and may further influence their choice of place of delivery and also lack some health services on time. According to this study, presence of premature ROM was also significantly associated with risk of having babies with adverse neonatal outcome. This study was found to be consistent with a study from Gambia, which reported higher risk of developing adverse neonatal outcomes among women who had premature ROM(33) Similarly this result was also consistent with a study from southern Ethiopia, which reported premature ROM to have a significant effect on adverse neonatal outcomes(29) This may be because of the risk of developing infection when the duration of rupture prolongs and a subsequent neonatal sepsis that may alter neonates APGAR score and may also need NICU admission.

Induction of labor was found to have a statistically significant effect on adverse neonatal outcomes in both short and recommended IPI mothers. This result was consistent with a study from suhul shire(21) This might be due to the risk of subsequent fetal distress after labor establishment through induction.

This study also showed that, C/S delivery was associated with higher risk of having adverse neonatal outcomes than vaginal delivery. This result was consistent with study from low and middle income countries(34) that showed delivery through $\mathrm{C} / \mathrm{S}$ to be associated with adverse neonatal outcomes. This might be due to the fact that mothers for whom $\mathrm{C} / \mathrm{S}$ done would have fetal distress during labor as an indication for $\mathrm{C} / \mathrm{S}$ and this subsequently affects the neonatal condition.

\section{Limitation Of The Study}

Cross-sectional nature of this study limits to set a causal-effect relationship between dependent and independent variables. Selection bias might be also the limitation of the study.

Mothers who deliver in health centers in Awi zone were not included, due to the lack of some relevant activities such as obstetric ultrasound, to estimate the GA and absence of NICU.

\section{Conclusion}

There was significant difference in proportion of adverse neonatal outcomes among short and recommended IPI mothers, $37.1 \%$ and $20.6 \%$ respectively. Urban residency and vaginal delivery were significantly associated with less risk of adverse neonatal outcomes among mothers in the short IPI groups. Whereas urban residency, intended pregnancy status, spontaneous labor initiation and absence of ROM before labor were reported as a protective for adverse neonatal outcomes in recommended IPI 
mothers. Provision of proper health service coverage at rural area and minimizing $\mathrm{C} / \mathrm{S}$ rate to reduce adverse neonatal outcome is highly recommended.

\section{Abbreviations}

ACOG American College of Obstetrics and Gynecology

ANC Antenatal Care

APGAR Appearance Pulse Grimace Activity Respiration

C/S Cesarean Section

Cl Confidence Interval

EDHS Ethiopian Demographic Health Survey

GA Gestational Age

IPI Interpregnancy Interval

LBW Low Birth Weight

NICU Neonatal Intensive Care Unit

ROM Rupture of Membrane

USAID United States Agency for International Development

WHO World Health Organization

\section{Declarations}

\section{Ethics approval and consent to participate}

Ethical clearance was obtained from the institutional review board of Bahir Dar University.

Formal letter of cooperation was written for public hospitals in Awi Zone, dangla primary hospital, Injibara general hospital, Jawi, Gimjabet and Chagni primary hospitals and permission was obtained. Written consent was obtained from each study participants. Confidentiality of information and privacy was maintained.

Consent for publication: Not applicable

Availability of data and materials: The datasets used and/or analyzed during the current study is available from the corresponding author on reasonable request. 


\section{Competing Interests}

The authors declare that they have no competing interests.

Funding: Bahir Dar University. This study was done by the fund obtained from Bahir Dar University for the design and collection, analysis and interpretation of data. However, the funder has no specific role in the preparation of the manuscript.

\section{Authors' Contribution}

JCF conceived and designed the protocol, performed the data collection, contributed for data analysis, wrote the paper and revised the manuscript.

AM, KGW and TW participate on the editorial and data entry and analysis. All authors read and approved the final paper.

\section{Acknowledgments}

The authors would like to pass their gratitude to Bahirdar University for the approval of ethical clearance. We would like to extend our thanks to Awi zone public hospitals for permitting to conduct the study and providing the necessary preliminary information. The authors are also very grateful for data collectors and mothers who participated in this study.

\section{References}

1. Ramos D, Stuebe A, Blackwell SC. Interpregnancy Care. 2019.

2. Gebremedhin AT, Regan AK, Malacova E, Marinovich ML, Ball S, Foo D, et al. Effects of interpregnancy interval on pregnancy complications: protocol for systematic review and metaanalysis. BMJ open. 2018;8(8):e025008.

3. Mignini L, Carroli G, Betran A, Fescina R, Cuesta C, Campodonico L, et al. Interpregnancy interval and perinatal outcomes across Latin America from 1990 to 2009: a large multi-country study. BJOG: An International Journal of Obstetrics Gynaecology. 2016;123(5):730-7.

4. Organization WH. World health statistics-Monitoring health for the SDGs, sustainable development goals. Geneva: World Health Organization; 2017. Licence: CC BY-NC-SA 3.0 IGO. 2017.

5. Koh HK. A 2020 vision for healthy people. N Engl J Med. 2010;362(18):1653-6.

6. Lassi ZS, Majeed A, Rashid S, Yakoob MY, Bhutta ZA. The interconnections between maternal and newborn health-evidence and implications for policy. The Journal of Maternal-Fetal Neonatal Medicine. 2013;26(sup1):3-53.

7. Appareddy S, Pryor J, Bailey B. Inter-pregnancy interval and adverse outcomes: Evidence for an additional risk in health disparate populations. The Journal of Maternal-Fetal Neonatal Medicine. 2017;30(21):2640-4. 
8. UNICEF-WHO. United Nations Children's Fund and World Health Organization. Low birthweight: country, regional and global estimates. UNICEF New York; 2004.

9. Liu L, Oza S, Hogan D, Perin J, Rudan I, Lawn JE, et al. Global, regional, and national causes of child mortality in $2000-13$, with projections to inform post-2015 priorities: an updated systematic analysis. The Lancet. 2015;385(9966):430-40.

10. Yee $L$, Truong $Y$, Caughey A, Cheng $Y$. The association between interdelivery interval and adverse perinatal outcomes in a diverse US population. J Perinatol. 2016;36(8):593-7.

11. Van Eijsden M, Smits LJ, Van der Wal MF, Bonsel GJ. Association between short interpregnancy intervals and term birth weight: the role of folate depletion. Am J Clin Nutr. 2008;88(1):147-53.

12. Hanley GE, Hutcheon JA, Kinniburgh BA, Lee L. Interpregnancy interval and adverse pregnancy outcomes. Obstetrics Gynecology. 2017;129(3):408-15.

13. Männistö J, Bloigu A, Mentula M, Gissler M, Heikinheimo O, Niinimäki M. Interpregnancy interval after termination of pregnancy and the risks of adverse outcomes in subsequent birth. Obstetrics Gynecology. 2017;129(2):347-54.

14. Adam I, Ismail MH, Nasr AM, Prins MH, Smits LJ. Low birth weight, preterm birth and short interpregnancy interval in Sudan. The Journal of Maternal-Fetal Neonatal Medicine. 2009;22(11):1068-71.

15. Mahande MJ, Obure J. Effect of interpregnancy interval on adverse pregnancy outcomes in northern Tanzania: a registry-based retrospective cohort study. BMC Pregnancy Childbirth. 2016;16(1):140.

16. EDHS ED. Health Survey. Key indicators report. 2016.

17. mini Ethiopian Demographic and Health Survey. Mini Demographic and Health Survey. 2019.

18. Abdo R, Endalemaw T, Tesso F. Prevalence and associated Factors of Adverse Birth Outcomes amongWomen Attended Maternity Ward at Negest Elene Mohammed MemorialGeneral Hospital in Hosanna Town, SNNPR, Ethiopia. J Women's Health Care. 2016;5(4).

19. Kassahun EA, Zeleke LB, Dessie AA, Gersa BG, Oumer HI, Derseh HA, et al. Factors associated with unintended pregnancy among women attending antenatal care in Maichew Town. Northern Ethiopia 2017 BMC research notes. 2019;12(1):381.

20. Gebremeskel F, Gultie T, Kejela G, Hailu D, Workneh Y. Determinants of adverse birth outcome among mothers who gave birth at hospitals in Gamo Gofa Zone, Southern Ethiopia: a facility based case control study. Quality in Primary Care. 2017;25(5):259-66.

21. Adhena T, Haftu A, Gebre G, Dimtsu B. Assessment of Magnitude and Associated Factors of Adverse Birth Outcomes among Deliveries at Suhul Hospital Shire, Tigray, Ethiopia From September, 2015 to February, 2016. Res Rev J Med Sci Technol. 2017;6(1):1-10.

22. Kassa GM, Arowojolu A, Odukogbe A, Yalew AW. Adverse neonatal outcomes of adolescent pregnancy in Northwest Ethiopia. PloS one. 2019;14(6).

23. Kassahun EA, Mitku HD, Getu MA. Adverse birth outcomes and its associated factors among women who delivered in North Wollo zone, northeast Ethiopia: a facility based cross-sectional study. BMC 
Research Notes. 2019;12(1):357.

24. Adane AA, Ayele TA, Ararsa LG, Bitew BD, Zeleke BM. Adverse birth outcomes among deliveries at Gondar University hospital, Northwest Ethiopia. BMC Pregnancy Childbirth. 2014;14(1):90.

25. Organization WH. Report of a WHO technical consultation on birth spacing: Geneva, Switzerland 1315 June 2005. World Health Organization, 2007.

26. Yesuf AEF, Hagos S, Assefa M. Effect of Interpregnancy Interval on Low Birth Weight in Gondar and Bahir Dar Referral Hospital: A Case Control Study from North West Ethiopia Journal of Health, Medicine and Nursing. 2016;31.

27. Tekelab T, Chojenta C, Smith R, Loxton D. Incidence and determinants of neonatal near miss in south Ethiopia: a prospective cohort study. BMC Pregnancy Childbirth. 2020;20(1):1-13.

28. Bener A, Saleh NM, Salameh KMK, Basha B, Joseph S, Samson N, et al. The impact of the interpregnancy interval on birth weight and other pregnancy outcomes. Revista Brasileira de Saúde Materno Infantil. 2012;12(3):233-41.

29. Tsegaye B, Kassa A. Prevalence of adverse birth outcome and associated factors among women who delivered in Hawassa town governmental health institutions, south Ethiopia, in 2017. Reproductive health. 2018;15(1):193.

30. Wolde HF, Tsegaye AT, Sisay MM. Late initiation of antenatal care and associated factors among pregnant women in Addis Zemen primary hospital, South Gondar, Ethiopia. Reproductive health. 2019;16(1):73.

31. Gebrekidan K, Worku A. Factors associated with late ANC initiation among pregnant women in select public health centers of Addis Ababa, Ethiopia: unmatched case-control study design. Pragmatic observational research. 2017;8:223.

32. Workie $\mathrm{H}$. adverse neonatal outcomes and associated risk factors in public and private hospitals of Mekelle city, Tigray, Ethiopia: Unmatched case-control study. Journal of pediatrics Therapeutics. 2013;08:39.

33. Jammeh A, Sundby J, Vangen S. Maternal and obstetric risk factors for low birth weight and preterm birth in rural Gambia: a hospital-based study of 1579 deliveries. Open Journal of Obstetrics Gynecology. 2011;1(03):94.

34. Harrison MS, Pasha O, Saleem S, Ali S, Chomba E, Carlo WA, et al. A prospective study of maternal, fetal and neonatal outcomes in the setting of cesarean section in low-and middle-income countries. Acta obstetricia et gynecologica Scandinavica. 2017;96(4):410-20.

35. Annex I. English version questionnaire.

\section{Supplementary Files}

This is a list of supplementary files associated with this preprint. Click to download.

- Englishversionquestionnaire.docx 